\title{
Iron Deposition Leads to Neuronal $\alpha$-Synuclein Pathology by Inducing Autophagy Dysfunction
}

\author{
Wenbin Wan ${ }^{1 \dagger}$, Lirong Jin ${ }^{1 * \dagger}$, Zigao Wang, ${ }^{1,2}$, Lingyan Wang ${ }^{3}$, Guoqiang Fei', Fanlong Ye', \\ Xiaoli Pan ${ }^{1}$, Changpeng Wang ${ }^{1}$ and Chunjiu Zhong ${ }^{1 *}$ \\ ${ }^{1}$ Department of Neurology, Zhongshan Hospital, Fudan University, Shanghai, China, ${ }^{2}$ Department of Neurology, Jingshan \\ Hospital, Fudan University, Shanghai, China, ${ }^{3}$ Experimental Research Center, Zhongshan Hospital, Fudan University, \\ Shanghai, China
}

Growing evidence has indicated that iron deposition in the substantia nigra plays an important role in Parkinson's disease (PD). However, the underlying mechanism is still elusive. Using primary dopaminergic neurons and SH-SY5Y cells cultured in vitro, we observed that iron loading increased $\alpha$-synuclein and reactive oxygen species (ROS) levels in these cells but did not affect the intracellular $\alpha$-synuclein mRNA levels.

Edited by:

Kathleen A. Maguire-Zeiss, Georgetown University, USA

Reviewed by: Jiajie Diao, University of Cincinnati, USA Timo T. Myöhänen, University of Helsinki, Finland

${ }^{*}$ Correspondence: Lirong Jin jin/r99@163.com;

Chunjiu Zhong zhongcj@163.com

tThese authors have contributed equally to this work.

Specialty section:

This article was submitted to

Neurodegeneration,

a section of the journal

Frontiers in Neurology

Received: 05 October 2016 Accepted: 04 January 2017 Published: 16 January 2017

Citation:

Wan W, Jin L, Wang Z, Wang L, Fei G, Ye F, Pan X, Wang C and Zhong C (2017) Iron Deposition Leads to Neuronal $\alpha$-Synuclein Pathology by Inducing Autophagy Dysfunction.

Front. Neurol. 8:1. doi: 10.3389/fneur.2017.00001 Furthermore, iron loading significantly downregulated Beclin-1 levels and decreased the ratio of microtubule-associated protein 1 light chain 3 isoforms (LC3 II/LC3 I). However, a significant change in the levels of autophagy-related gene 5 (Atg5) was not observed in either neurons or SH-SY5Y cells after iron treatment. After treatment with rapamycin, the iron loading-induced increase in the $\alpha$-synuclein level was significantly reversed and ROS generation was alleviated in both cultured neurons and SH-SY5Y cells. These results indicate that the inhibition of autophagy is critical for the pathological alterations in $\alpha$-synuclein induced by iron loading. Moreover, treatment with vitamin $\mathrm{E}$ did not affect the increase in the $\alpha$-synuclein levels but significantly eliminated the iron-induced ROS production. Together, our study shows that autophagy dysfunction contributes to iron-induced $\alpha$-synuclein pathology.

Keywords: Parkinson's disease, $\alpha$-synuclein, iron, autophagy, reactive oxygen species

\section{INTRODUCTION}

Parkinson's disease (PD) is one of the most common movement disorders. PD is clinically characterized by resting tremor, rigidity, and bradykinesia and is pathologically characterized by the aberrant accumulation of $\alpha$-synuclein and dopaminergic neuron loss in the substantia nigra pars compacta (1-3). The etiology and pathogenesis of PD are still largely unknown, and an effective therapy against disease progression has not been achieved. Thus, more efforts are needed to characterize the pathological etiology of PD.

Since Lhermitte's first report in 1924 that iron content was increased in the midbrain of individuals with PD (4), the role of iron deposition in PD progression has received substantial attention (5-9). As described in previous studies by our and other groups, an elevated nigral iron level is correlated with the disease severity in patients with $\operatorname{PD}(7,10)$. Accumulating evidence from in vitro studies has revealed that the expression of human $\alpha$-synuclein is modified by iron deposition, which subsequently results in the aggregation and toxicity of $\alpha$-synuclein $(11,12)$. Furthermore, an iron 
chelation treatment was shown to benefit patients with PD; substantia nigra (SN) iron levels were decreased and clinical and radiological improvements were observed (13). Many other studies have also indicated that elevated iron levels in the SN present a tractable target for PD $(14,15)$. Together, these results indicate that the aberrant accumulation of iron is crucial in PD progression. However, the mechanism underlying the iron-induced development of PD is still elusive.

Oxidative stress has been demonstrated to be an important factor in iron-induced pathologies, serving as a link between iron deposition and PD $(16,17)$. Elevated iron levels in the SN lead to the generation of reactive oxygen species (ROS), such as superoxide anion radicals and hydroxyl radicals; the increase in ROS level subsequently results in the aberrant upregulation of $\alpha$-synuclein and damage to dopaminergic neurons in $\operatorname{PD}(16,17)$. In the presence of iron, the highly reactive hydroxyl radical is produced by the Fenton reaction (16). As one of the most harmful ROS, this highly reactive hydroxyl radical damages proteins, nucleic acids, and the lipid membrane, resulting in cell injury and death (16). Nevertheless, controversy still exists. Oxidative stress is not considered a determining factor of the aberrant aggregation of $\alpha$-synuclein in PD. Radical eliminators thoroughly remove the generated ROS in vitro but are unable to entirely reverse the iron-induced upregulation of $\alpha$-synuclein (18). Moreover, anti-oxidants have little effect on improving the symptoms of PD (19-21). Thus, other potential mechanisms might be involved in the development of PD.

Autophagy is a degradation process that plays a pivotal role in the cellular energy balance and in eliminating misfolded proteins in cells (22). Autophagy is classified as macroautophagy, microautophagy, and chaperone-mediated autophagy (CMA) $(23,24)$. In the last decade, growing evidence has indicated an intimate relationship between autophagy and $\mathrm{PD}$, showing that autophagy dysregulation may play a critical role in the development of PD. Aggregates of $\alpha$-synuclein are mainly degraded via the autophagy pathway (25-27), including macroautophagy and CMA pathways (24). The autophagy signaling pathway has been reported to be disrupted in PD (28). Furthermore, the inhibition of autophagy results in the pathological accumulation of $\alpha$-synuclein in neurons (28). However, the role of autophagy in iron-induced PD pathology remains unclear.

In this study, we investigated the effect of iron deposition on neural cells and then evaluated the role of autophagy in the pathological, iron-induced accumulation of $\alpha$-synuclein.

\section{MATERIALS AND METHODS}

\section{Cell Culture and Treatments}

SH-SY5Y cells (Type Culture Collection of the Chinese Academy of Sciences, Shanghai, China) were cultured in Dulbecco's modified Eagle's medium (DMEM, Gibco, USA) supplemented with $10 \%$ fetal bovine serum (Gibco, USA), $100 \mathrm{U} / \mathrm{ml}$ penicillin, and $100 \mu \mathrm{g} / \mathrm{ml}$ streptomycin (Gibco, USA) at $37^{\circ} \mathrm{C}$ in a humidified atmosphere containing $5 \% \mathrm{CO}_{2}$. The cells were subcultured every 3 days and were grown to $70-80 \%$ confluence prior to treatment.
The primary mesencephalic cells were cultured using a previously described method (29) with several modifications. Briefly, on day 17, pregnant Sprague-Dawley (SD) rats were anesthetized, and the fetuses were collected to isolate and digest the cells. The mesencephalic dopaminergic region was retrieved. Cell suspensions were filtered, centrifuged, and plated onto poly-L-lysinecoated dishes at a density of $5 \times 10^{4}$ cells $/ \mathrm{cm}^{2}$. Approximately $5 \mathrm{~h}$ later, the medium was replaced with neurobasal medium supplemented with B27 and GlutaMAX (Invitrogen, USA). The cells were maintained for 12 days before administering the indicated treatment. This procedure used to collect primary cells from SD rats employed in this study was approved by the Medical Experimental Animal Administrative Committee of Zhongshan Hospital, Fudan University.

Cells were treated with ferrous chloride (Sigma, USA) prepared in sterilized water containing $0.01 \mathrm{~N} \mathrm{HCl}$ as previously described (30). Rapamycin (InvivoGen, USA) was dissolved in DMSO (Sigma, USA) as a $10 \mathrm{mM}$ sterile stock solution, and $0.2 \mu \mathrm{M}$ was employed as the working concentration of rapamycin, according to the manufacturer's instructions and previously published work $(31,32)$. Vitamin E (Sigma, USA) was used as an ROS scavenger as previously described $(18,33)$. The cells were incubated with the indicated agents for $24 \mathrm{~h}$ and then were harvested for detection.

\section{Knockdown of $\alpha$-Synuclein with Small Interfering RNAs (siRNAs)}

Cholestenone-modified siRNAs targeting rat $\alpha$-synuclein 5'-CCTCTATGTAGGTTCCAAA-3' was synthesized by BioTend (China). Twenty-four hours before the iron treatment, the neurons were transfected with the siRNAs using the Lipofectamine ${ }^{\circledR}$ RNAiMAX Transfection Reagent (Invitrogen, USA) according to the manufacturer's instructions.

\section{Measurement of Cell Viability}

Cell viability was measured using a CKK-8 assay according to the manufacturer's instructions (Dojindo, Japan). Three hours after the CCK- 8 solution was added, the absorbance was determined at $450 \mathrm{~nm}$ using a microplate reader (Thermo Fisher, USA).

\section{Immunofluorescent Staining}

Cells were cultured on a round slide. After treatment, the slide was washed with $0.01 \mathrm{M} \mathrm{PBS}$ and fixed with $4 \%$ PFA as previously described (34). The primary antibody (Mouse anti- $\alpha$-synuclein: BD, USA; Rabbit anti-NeuN: Abcam, USA) was added and incubated with the cells for $48 \mathrm{~h}$ at $4^{\circ} \mathrm{C}$. Then, the slide was exposed to the Alexa Fluor ${ }^{\circledR}$ antibody (Invitrogen, USA) and incubated for $1 \mathrm{~h}$ at $37^{\circ} \mathrm{C}$. The nucleic acids were stained with DAPI (Invitrogen, USA). Following a final wash and mounting with anti-fade medium (Sigma, USA), images were acquired using a fluorescence microscope (Nikon, Japan). The fluorescence intensity was determined using Image-Pro Plus, Version 6.0 (MediaCybernetics, Inc., USA).

\section{Western Blots}

Western blotting was conducted as previously described (35, 36). After an incubation with the indicated antibodies (Beclin-1, 
Atg5, and LC3 I/II: CST, USA; $\alpha$-synuclein: BD Biosciences, USA; GAPDH: Santa Cruz Biotechnology, USA), the membranes were analyzed, and images were captured using an Odyssey infrared fluorescence imaging system (LI-COR, USA).

\section{Real-time qPCR}

Quantitative PCR was conducted using our previously described method (36), and the $2^{-\Delta \Delta C T}$ method was used to analyze the fold change in the levels of the $\alpha$-synuclein mRNA. The forward and reverse sequences of the PCR primers are listed in Table $\mathbf{1 .}$

\section{Measurement of Intracellular ROS Levels}

The levels of oxidative stress were evaluated using an ROS detection kit (Invitrogen, USA), and the procedure was conducted according to the manufacturer's instructions. The relative fluorescence intensity of the cells was quantified using a multi-detection microplate reader (Bio-Rad, USA) at an excitation wavelength of $488 \mathrm{~nm}$ and an emission wavelength of $525 \mathrm{~nm}$. The intracellular ROS levels were expressed as a percentage of the control cells.

\section{Transmission Electron Microscopy (TEM)}

Transmission electron microscopy was performed using a previously described method (37). Briefly, the cells were pre-fixed with ice-cold $2.5 \%$ glutaraldehyde (Sigma, USA) diluted in $0.1 \mathrm{M}$ phosphate-buffered saline and post-fixed with $1 \%$ osmium tetroxide buffer. After dehydration in a gradient series of ethyl alcohol, the cells were embedded in epoxy resin. Ultrathin sections (60-nm thick) were stained with uranyl acetate and lead citrate and examined using a transmission electron microscope (Philips CM120, the Netherlands).

\section{Statistical Analysis}

All results are expressed as the means \pm SD. Statistical analyses were performed using GraphPad Prism 5.0 software (GraphPad Software, Inc., USA). All experiments were independently repeated three times. The statistical significance of the differences among different groups was analyzed using one-way analysis of variance or Student's $t$-test, in which $p<0.05$ was considered significant.

\section{RESULTS}

\section{Iron Decreased Cell Viability}

As shown in Figure 1, primary cultured neurons and SH-SY5Y cells exhibited alterations in cell viability following incubation with different concentrations of ferrous chloride. After a 24-h

\begin{tabular}{|c|c|c|c|}
\hline \multirow{3}{*}{$\begin{array}{l}\text { Genes of interest } \\
\text { Human SCNA }\end{array}$} & \multirow{3}{*}{$\begin{array}{c}\text { Gene ID } \\
6622\end{array}$} & \multicolumn{2}{|c|}{ Sequence $\left(5^{\prime} \rightarrow 3^{\prime}\right)$} \\
\hline & & Forward & AAGAGGGTGTTCTCTATGTAGGC \\
\hline & & Reverse & GCTCCTCCAACATTTGTCACTT \\
\hline \multirow[t]{2}{*}{ Human $\beta$-actin } & 60 & Forward & CATGTACGTTGCTATCCAGGC \\
\hline & & Reverse & СTCCTTAATGTCACGCACGAT \\
\hline \multirow[t]{2}{*}{ Rat SCNA } & 29219 & Forward & AAGGGTACCCACAAGAGGGA \\
\hline & & Reverse & AACTGAGCACTTGTACGCCA \\
\hline \multirow[t]{2}{*}{ Rat $\beta$-actin } & 81822 & Forward & CATCCGTAAAGACCTCTATGCC \\
\hline & & Reverse & AGGATAGAGCCACCAATCCAC \\
\hline
\end{tabular}

treatment with $100 \mu \mathrm{M}$ ferrous chloride, the viability of neurons and SH-SY5Y cells was significantly reduced $(p<0.05)$. The viability of neurons and SH-SY5Y cells exhibited a decreasing trend that was not significantly different $(p>0.05)$ after incubation with $50 \mu \mathrm{M}$ ferrous chloride. Incubation with $20 \mu \mathrm{M}$ ferrous chloride did not reduce the viability of neurons and SH-SY5Y cells. Thus, we employed $20 \mu \mathrm{M}$ ferrous chloride in the subsequent experiments.

\section{Iron Increased $\alpha$-Synuclein Levels and ROS Generation but Did Not Affect the Expression of $\alpha$-Synuclein mRNA}

To determine whether iron deposition affects $\alpha$-synuclein levels, we evaluated the effect of iron on the changes in the $\alpha$-synuclein levels in primary neurons and SH-SY5Y cells. Using immunofluorescence staining and Western blotting, we observed that iron exposure upregulated the levels of $\alpha$-synuclein protein (Figures 2A,B,D,E, $p<0.01$ for neurons, $p<0.05$ for SH-SY5Y cells). However, the iron treatment did not affect the levels of $\alpha$-synuclein mRNA (Figure 2C, $p>0.05$ ).

Consistent with the results from previous studies (38), we also observed a 2.6-fold increase in ROS levels in iron-treated cells compared with those of the control (Figure 5A, $p<0.05$ ).

\section{Iron Inhibited Autophagy}

Alterations in the levels of the autophagy-related proteins in both of primary neurons and SH-SY5Y cells, including Beclin1, autophagy-related gene 5 (Atg5), and microtubule-associated protein 1 light chain 3 isoforms (LC3 I/LC3 II), were examined by Western blotting. The iron treatment significantly downregulated the levels of Beclin1 (Figures 3A,B, $p=0.01$ for neurons, $p=0.02$ for SH-SY5Y cells). Moreover, the levels of LC3 II and the LC3 II/I ratio were also significantly decreased (Figures $3 \mathbf{A , B}, p=0.01$ for neurons, $p=0.03$ for SH-SY5Y cells). However, Atg5 levels were not significantly reduced in response to the iron treatment (Figures 3A,B, $p>0.05$ ).

Using TEM, we consistently confirmed that the number of autophagosomes in the iron-treated neurons was less than the number in control neurons or SH-SY5Y cells (Figure 3C).

\section{Rapamycin Alleviated Iron-Induced $\alpha$ - Synuclein Accumulation and ROS Generation}

To further investigate the role of autophagy inhibition in ironinduced pathological change in our in vitro work, we employed an autophagy activator, rapamycin. Following co-incubation with or without iron, rapamycin was observed a slight trend of reduction but did not significantly affect the $\alpha$-synuclein levels in the control groups (Figures 4A,C,D, $p>0.05$ ); however, the upregulation of the $\alpha$-synuclein levels induced by iron was ameliorated by the rapamycin treatment (Figures $\mathbf{4 A}, \mathbf{C}, \mathbf{D}, p<0.05$ ).

We then evaluated the levels of oxidative stress in rapamycintreated neurons. As shown in Figure 4, rapamycin potently eliminated iron-induced ROS generation (Figures $\mathbf{4 G , H}, p<0.01$ ) as compared with the iron treatment alone. 

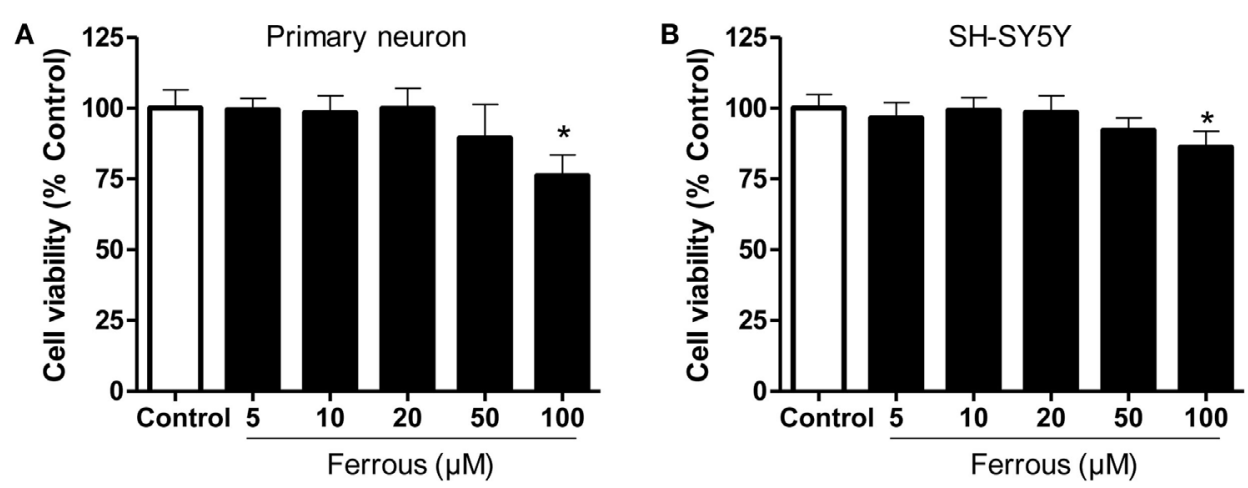

FIGURE 1 | Effect of iron on cell viability. The effects of different concentrations of iron on cell viability were determined using the CCK-8 assay. Iron concentrations greater than $50 \mu \mathrm{M}$ led to significant cell damage. Iron concentrations greater than $100 \mu \mathrm{M}$ induced marked toxicity to both primary neurons (A) and SH-SY5Y cells (B). The results are presented as means \pm SD, and one-way analysis of variance was employed to determine the statistical significance of the differences. ${ }^{*} p<0.05$ vs. the control.

A

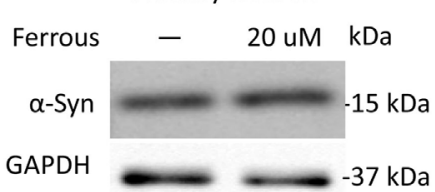

SH-SY5Y

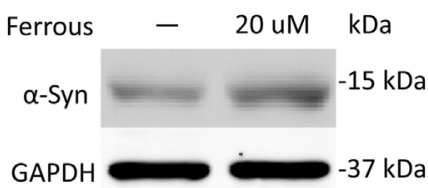

D

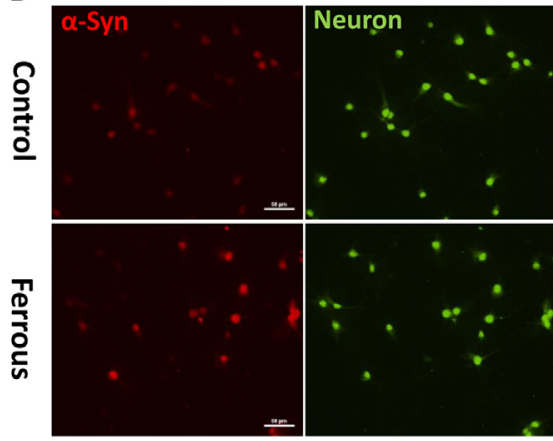

B

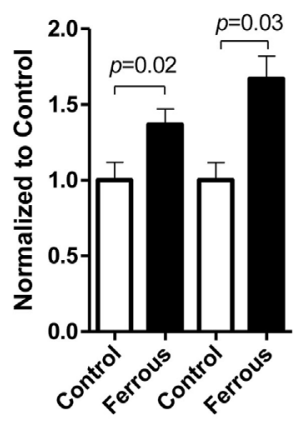

Primary Neuron SH-SY5Y
C

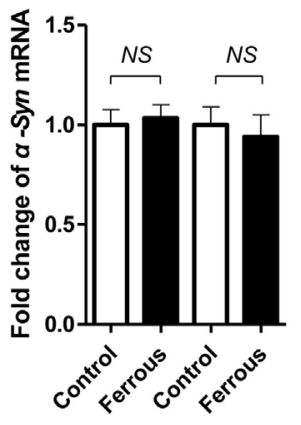

Primary Neuron SH-SY5Y

E

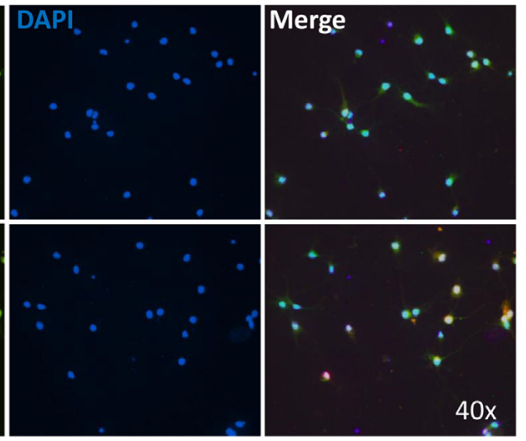

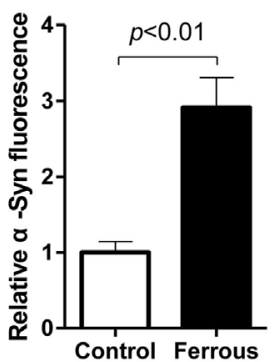

FIGURE 2 | Iron increased the levels of the $\alpha$-synuclein protein but did not affect the expression of the $\alpha$-synuclein mRNA. After a 24 -h iron treatment, cells were harvested to examine the protein and mRNA levels. As shown above, the iron treatment increased the level of the $\alpha$-synuclein protein $[\alpha-S y n(\mathbf{A}, \mathbf{D})$, $p<0.05]$ but did not affect the levels of the $\alpha$-Syn mRNA [(C), $p>0.05]$. (B,E) show the quantitation of the data shown in (A,D), respectively. The results are presented as means $\pm \mathrm{SD}$, and Student's $t$-test was used to determine the statistical significance of the differences.

\section{Vitamin E Eliminated Iron-Induced ROS Generation but Did Not Alleviate $\alpha$-Synuclein Pathology}

As mentioned above, the iron treatment increased the ROS levels in vitro, indicating that iron induced oxidative stress.
To determine whether the ROS eliminator vitamin E could influence the iron-induced oxidative stress and $\alpha$-synuclein pathology, we tested the effects of different concentrations of vitamin $\mathrm{E}$ on primary neurons. At concentrations ranging from 1 to $10 \mu \mathrm{M}$, the vitamin E treatment reduced iron-induced ROS generation in neurons (Figure $5 \mathbf{A}, p<0.05$ ). In the presence of $1 \mu \mathrm{M}$ vitamin $\mathrm{E}$, 

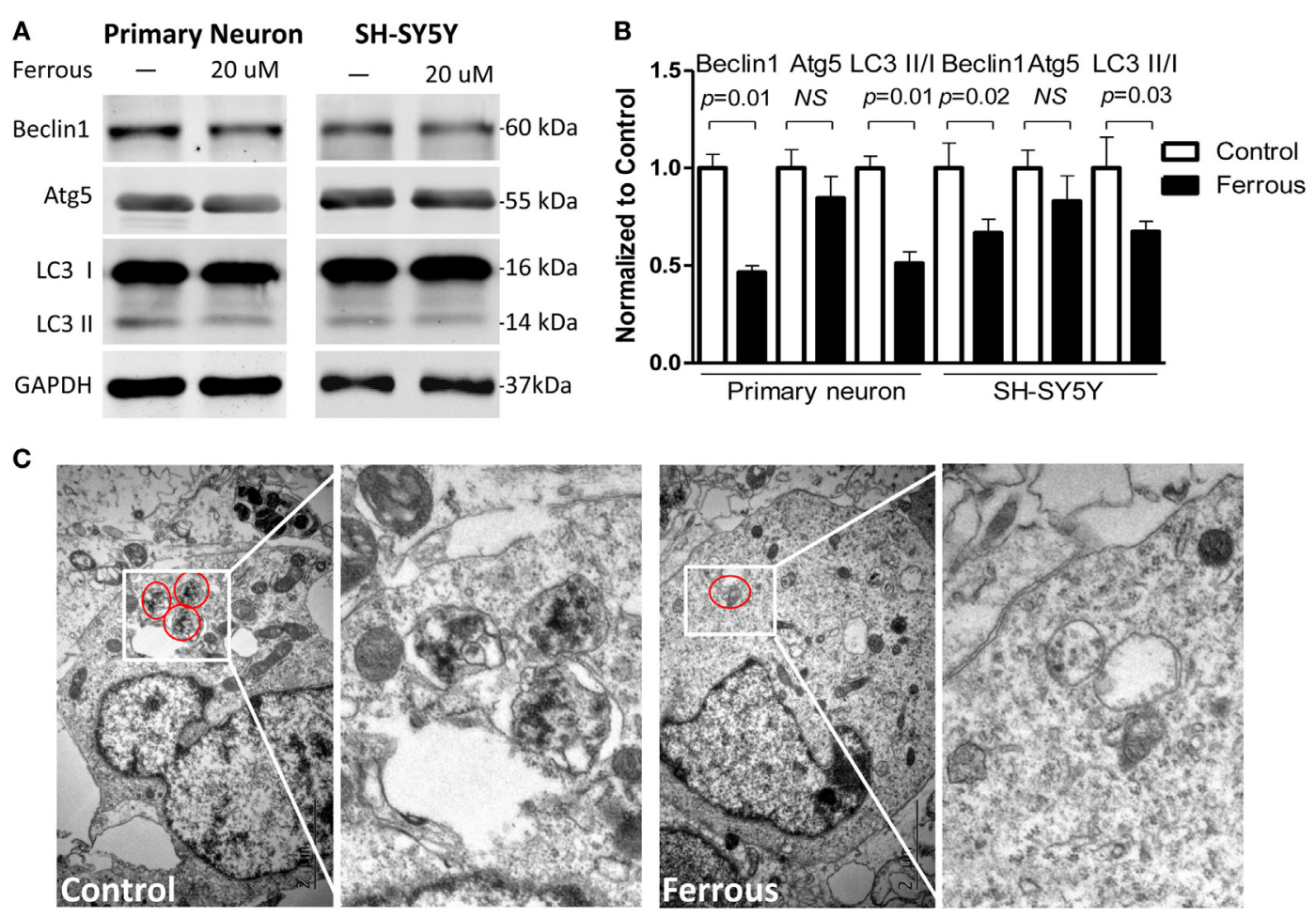

FIGURE 3 | Iron inhibited autophagy. The levels of the Beclin-1 $(p<0.05)$ and Atg5 proteins $(p>0.05)$ were decreased in iron-treated cultures (A). The LC3 II level was also decreased and the LC3II/I ratio was reduced in the iron-treated cells [(A), $p<0.05]$. (B) shows the quantitation of the results shown in (A). (C) shows the autophagosomes present in primary neurons. The results are presented as means $\pm \mathrm{SD}$, and Student's $t$-test was used to determine the statistical significance of the differences.

the ROS levels were significantly decreased compared with those of cells treated with iron alone and of the control (Figure 5A, $p=0.03$ ). However, following treatment with $2 \mu \mathrm{M}$ vitamin $\mathrm{E}$, the ROS levels were not significantly different from the control (Figure 5A, $p>0.05$ ).

Primary neurons were then treated with $20 \mu \mathrm{M}$ iron in the presence and absence of $2 \mu \mathrm{M}$ vitamin $\mathrm{E}$ to analyze the effect of oxidative stress on the elevated $\alpha$-synuclein levels. As shown in Figures 5B-E, incubation with vitamin $\mathrm{E}$ alone did not affect the $\alpha$-synuclein levels in neurons or the iron-induced upregulation of the $\alpha$-synuclein levels $(p>0.05)$. Although iron-induced oxidative stress was inhibited in vitro, the increased $\alpha$-synuclein levels were not reduced by vitamin $\mathrm{E}$.

\section{$\alpha$-Synuclein Silencing Did Not Affect the Iron-Induced Inhibition of Autophagy}

According to previous reports, the pathological changes in $\alpha$-synuclein, such as its overexpression and formation of oligomers, inhibit autophagy $(39,40)$. As shown in this work, iron deposition led to dysfunctional autophagy and $\alpha$-synuclein pathology. We then transfected neurons with siRNAs targeting $\alpha$-synuclein to determine whether the inhibition of autophagy was directly mediated by iron or by the increase in the level of the $\alpha$-synuclein protein. First, the $\alpha$-synuclein levels were determined in cells transfected with the siRNAs using Western blotting (Figure 6A, $p<0.01)$. Subsequently, we evaluated the changes in the levels of autophagic proteins including Beclin1, Atg5, and LC3 II/LC3 I in iron-treated neurons within siRNAs transfection. The ironinduced downregulation of the Beclin-1, Atg5, and LC3 II levels was not significantly changed by the silencing of $\alpha$-synuclein compared with the effects seen in the iron-treated neurons (Figures 6B-D, $p>0.05$ ).

\section{DISCUSSION}

The mechanisms underlying abnormal $\alpha$-synuclein aggregation in dopaminergic neurons in patients with PD are elusive and require further investigation. Iron accumulation in the brain has been implicated in PD (5-9), but our current understanding of this phenomenon is very limited. Substantial evidence suggests a robust link between iron deposition in the $\mathrm{SN}$ and the pathological lesions observed in patients with PD $(11,12,14,15)$. As shown in this study, iron-treated neuronal cells exhibited $\alpha$-synuclein aggregates and the autophagy signaling pathway was inhibited. Following an incubation with rapamycin, the iron-induced pathologies of $\alpha$-synuclein aggregation and ROS generation were alleviated by the activation of autophagy, suggesting a critical role for autophagy in PD.

Oxidative stress results from an imbalance between ROS generation and the intracellular detoxifying systems (16). Oxidative 

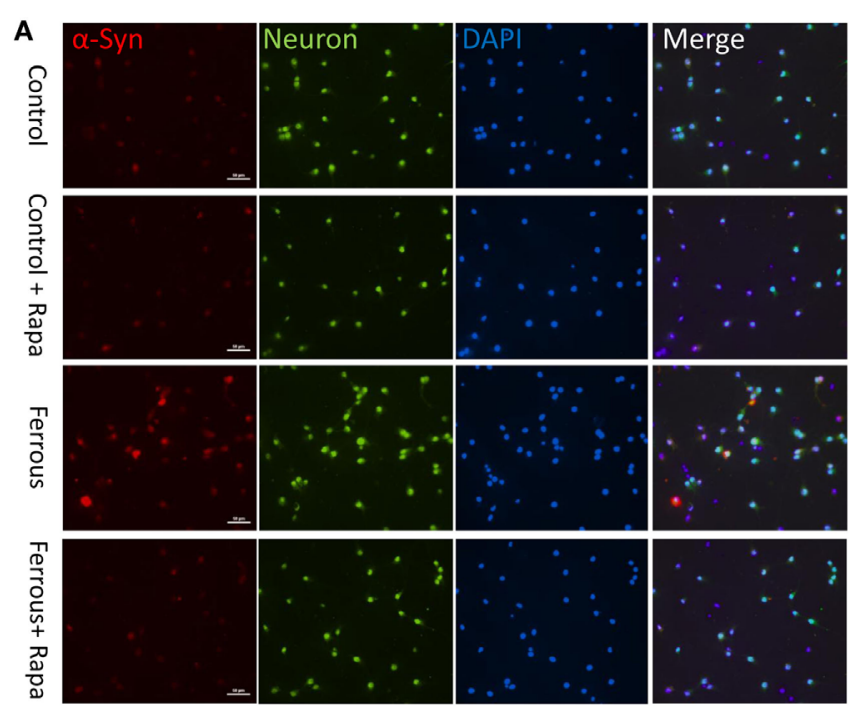

D

C

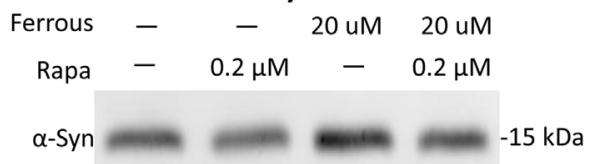

GAPDH

$-37 \mathrm{kDa}$

E
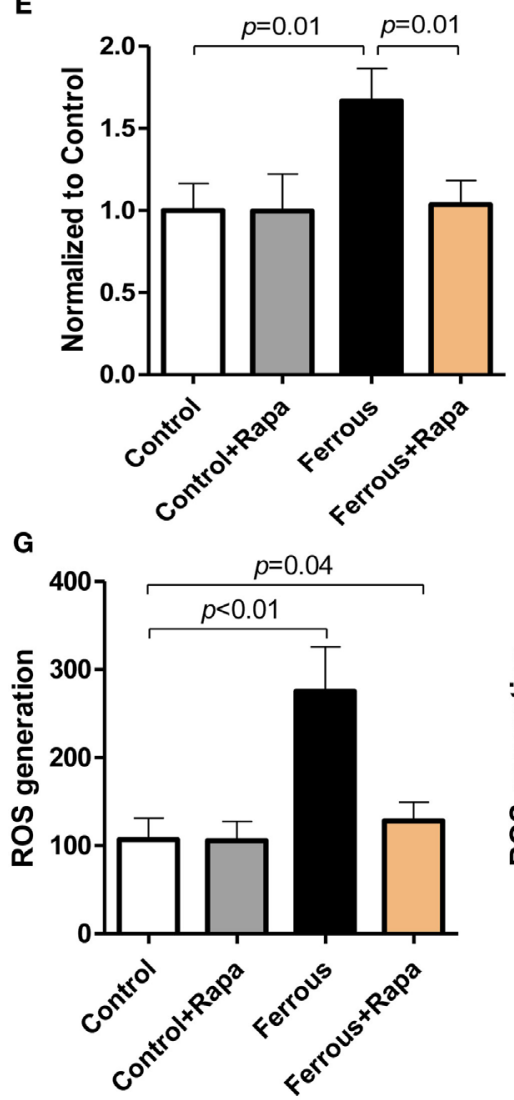

H

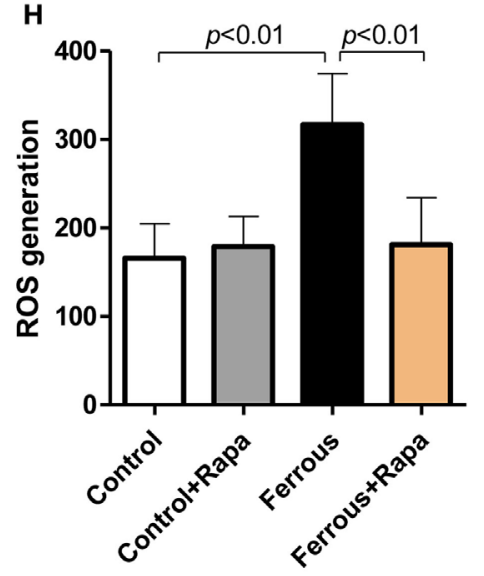

B

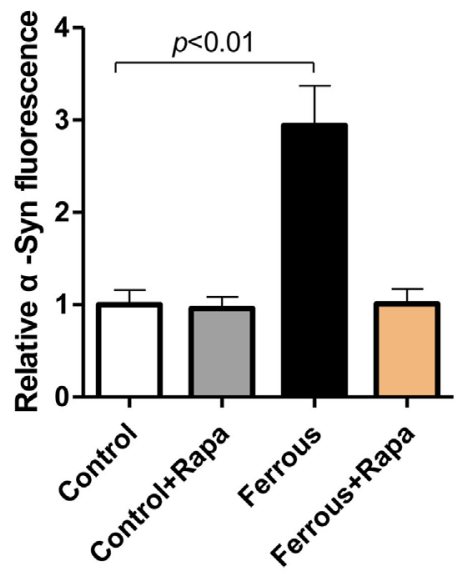

SH-SY5Y

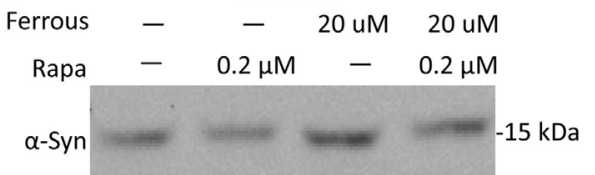

GAPDH $-37 \mathrm{kDa}$

F
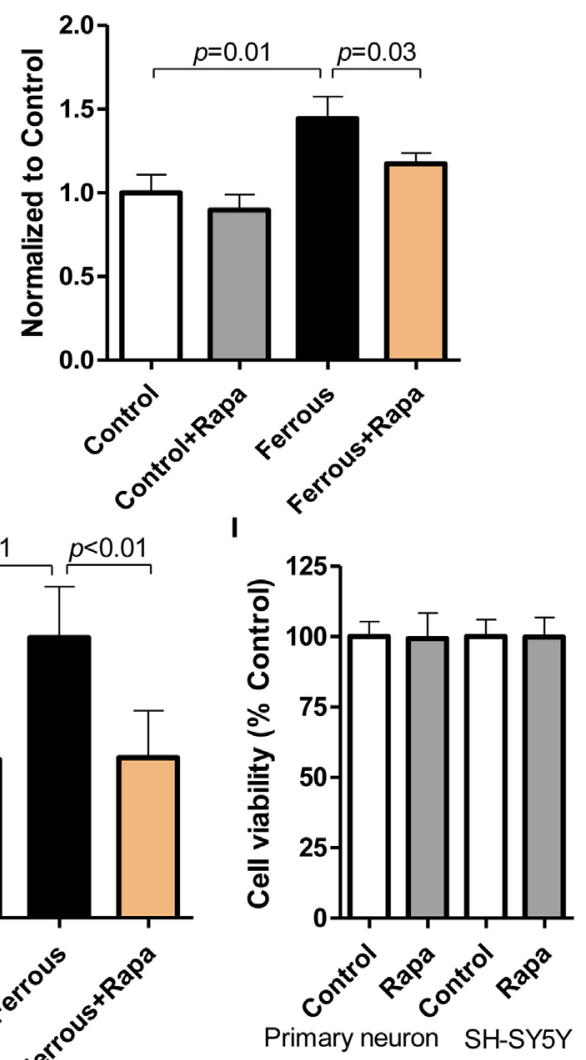
FIGURE 4 | Continued

Rapamycin alleviated the iron-induced pathological changes, including $\alpha$-synuclein accumulation and reactive oxygen species (ROS) production Rapamycin (Rapa) was used to activate autophagy in neurons. Compared with the iron treatment alone, Rapa significantly reversed the upregulation of $\alpha$-Syn $[(\mathbf{A}, \mathbf{C}, \mathbf{D}), p<0.05]$ and reduced ROS production in the cells $[(\mathbf{G}, \mathbf{H}), p<0.05] .(\mathbf{A}, \mathbf{C}, \mathbf{G})$ show the data obtained from primary neurons; $(\mathbf{D}, \mathbf{H})$ show the data obtained from SH-SY-5Y cells. (B,E,F) show the quantitation of the data shown in (A,C,D), respectively. (I) shows the effect of Rapa on cell viability performing that the concentration of $0.2 \mu \mathrm{M}$ did not affect cell viability in vitro $(p>0.05)$. The results are presented as means \pm SD, and one-way analysis of variance was employed to determine the statistical significance of the differences.
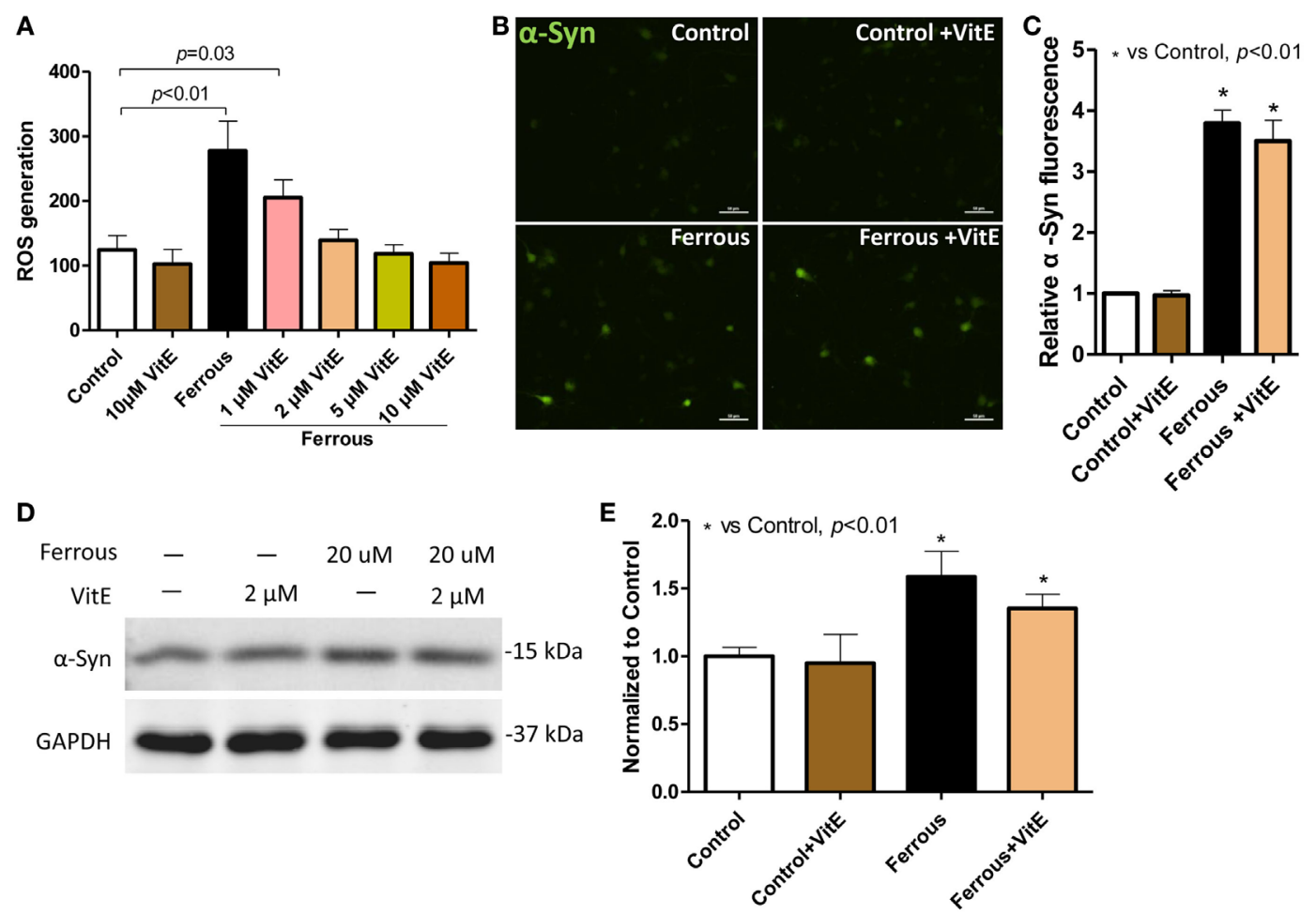

FIGURE 5 | Vitamin E eliminated reactive oxygen species (ROS) production but did not alleviate iron-induced $\alpha$-synuclein pathology in neurons. Vitamin E (Vit E), an ROS eliminator, was added to the cultures to evaluate the role of oxidative stress in iron-induced pathology in neurons. ROS production was completely eliminated by Vit E (A). However, the iron-induced increase in the $\alpha$-Syn levels was not reduced by the elimination of ROS production (B,D). (C,E) show the quantitation of the results presented in (B,D), respectively. The results are presented as means \pm SD, and one-way analysis of variance was employed to determine the statistical significance of the differences. ${ }^{* *} p<0.05$ vs. the control.

stress has been shown to be an important component of the ironinduced pathological changes, and some researchers posited that oxidative stress is a vital factor that contributes to $\alpha$-synuclein pathology in PD $(16,17)$. However, recent evidence has revealed that oxidative stress is not responsible for iron-induced lesions in PD (18), suggesting that the effects of oxidative stress on PD might require re-evaluation. We also achieved a similar result in vitro in this study, as the vitamin $\mathrm{E}$ treatment eliminated ROS but was unable to reverse the iron-induced upregulation of $\alpha$-synuclein. Our results are consistent with those of a previously published study by Li et al. who reported that the elimination of iron-induced ROS production only partially alleviated the intracellular $\alpha$-synuclein aggregation in SK-N-SH cells (18). In previous studies, the administration of anti-oxidant drugs did not confer any protective effects on attenuating the risk of PD (20).
Furthermore, a randomized clinical trial showed that a high dosage of anti-oxidants had a finite benefit for patients with PD (19). These results compelled us to examine the mechanisms underlying iron-induced PD-like pathology, in which other mechanisms may be involved.

Autophagy is an intracellular catabolic program that is active under normal and pathological conditions. It is executed by multiple autophagy-related proteins, including Beclin1, LC3I/II, Atg5, and others (41). This process degrades damaged proteins and organelles via the autophagy-lysosome pathway and ubiquitin-proteasome system, plays an essential role in the survival of cellular organisms, and provides the necessary materials for cells for cells to compensate for stress conditions such as starvation (41). Excessive activation of autophagy is considered pernicious and has been determined to contribute to neuronal death (42). 

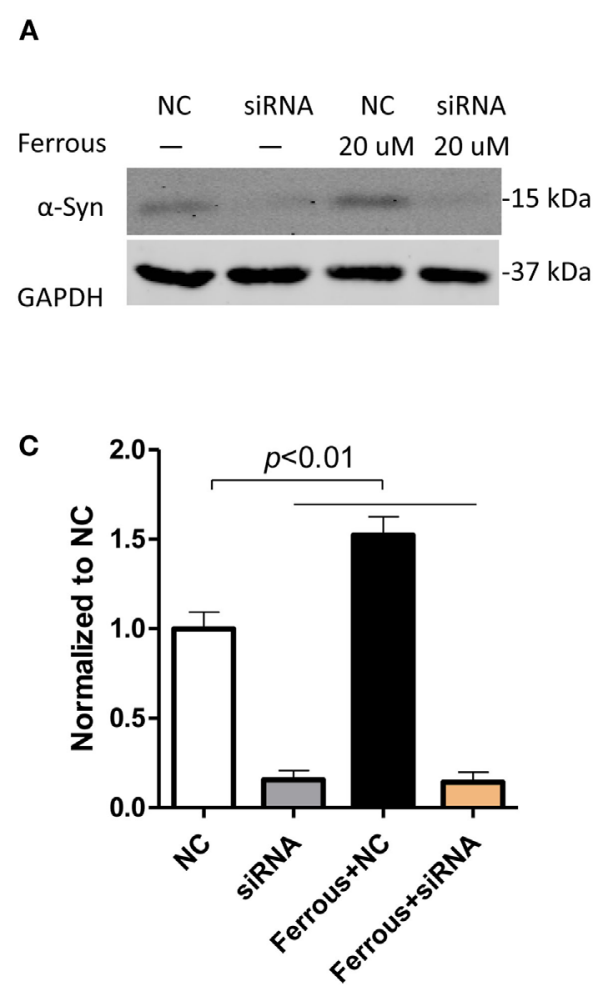

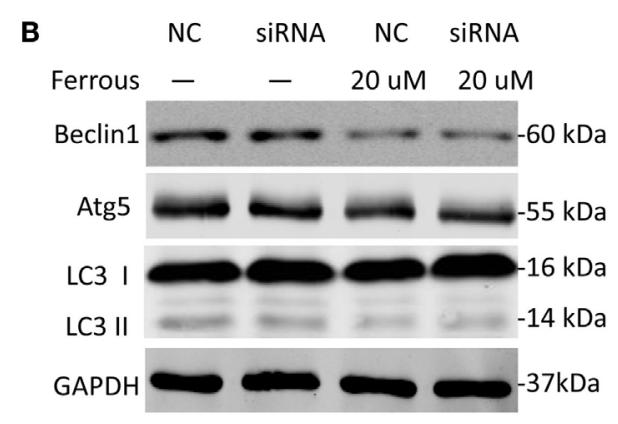

D

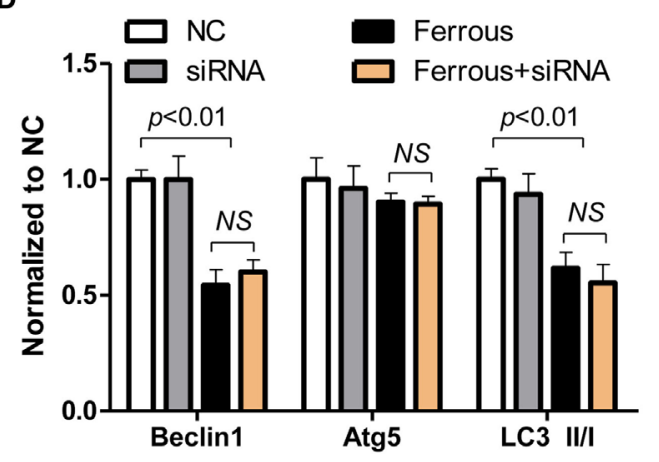

FIGURE 6 | Iron-induced inhibition of autophagy was not attenuated by $\boldsymbol{\alpha}$-synuclein silencing in neurons. The effect of small interfering RNAs (siRNAs) targeting $\alpha$-synuclein was determined using Western blotting (A). (B) shows that silencing of $\alpha$-synuclein did not affect iron-induced inhibition of autophagy. (C,D) show the quantitation of the results presented in $(\mathbf{A}, \mathbf{B})$, respectively. The results are presented as means \pm SD, and one-way analysis of variance was employed to determine the statistical significance of the differences. ${ }^{* *} p<0.05$ vs. the negative control (NC).

The disruption of autophagy is also harmful, resulting in the accumulation of misfolded proteins and dysfunctional organelles in cells $(43,44)$. The coordination of autophagic functions, including activation and inhibition, ensures the balance between cell growth and death. Thus, autophagy is not only critical for cellular survival and normal function but also plays dual roles in cellular life and death (45).

Numerous studies have indicated that autophagy is involved in the pathological changes observed in PD (25-27). However, the explicit role of autophagy in iron-induced pathology is still uncertain. As shown in this study, iron led to $\alpha$-synuclein pathology in neurons by disrupting Beclin-1-dependent autophagy. Although numerous signaling pathways, including oxidative stress, have been shown to contribute to the progression of PD, the pathogenesis of the disease remains to be clarified. Impaired autophagy has been shown to be involved in the pathological changes in $\alpha$-synuclein in PD (46-48). As shown in this study, iron loading resulted in $\alpha$-synuclein accumulation and impaired autophagy in both primary neurons and SH-SY5Y cells. The iron treatment significantly decreased the levels of the Beclin 1 and Atg5 proteins. In addition, the LC3 II level and LC II/I ratio were significantly decreased. Rapamycin significantly reversed the iron-induced upregulation of the neuronal $\alpha$-synuclein levels by activating the autophagy pathway. Furthermore, rapamycin also ameliorated iron-induced ROS generation in neurons, indicating that disruptions in autophagy were involved in iron-induced oxidative stress. Additionally, $\alpha$-synuclein silencing in primary neurons transfected with siRNAs did not affect the iron-induced inhibition of autophagy, suggesting that the iron treatment primarily disrupted autophagy but not the subsequent pathological changes in the $\alpha$-synuclein levels. Together, our data indicate that iron-induced autophagy dysfunction may be responsible for $\alpha$-synuclein accumulation. Moreover, iron deposition alone may directly inhibit the autophagy signaling pathway. However, we still cannot conclude that the pathological changes in $\alpha$-synuclein observed in this work do not affect the function of the autophagy pathway because the toxic effects of $\alpha$-synuclein, including the inhibition of autophagy, might be masked by the changes induced by the iron treatment.

In conclusion, autophagy is critical for the iron-induced pathogenesis of PD. Oxidative stress is also associated with $\alpha$-synuclein accumulation but may not play the key role in ironinduced $\alpha$-synuclein pathology in PD. Normally, autophagy is an important contributor to intracellular homeostasis; thus, maintaining proper activity of the autophagy pathway is essential for eliminating aberrant protein aggregates. Based on the data from this study, the regulation of autophagy is a potential therapeutic target for PD. However, further investigation is still needed to clarify the role of the autophagy pathway in PD, particularly in in vivo models and in patients. 


\section{AUTHOR CONTRIBUTIONS}

LJ and CZ designed the research; WW, ZW, LW, and FY performed the research; XP, CW, and GF analyzed the data; WW and LJ wrote the paper. All the authors approved the final version of the manuscript.

\section{REFERENCES}

1. Ganz J, Lev N, Melamed E, Offen D. Cell replacement therapy for Parkinson's disease: how close are we to the clinic? Expert Rev Neurother (2011) 11: 1325-39. doi:10.1586/ern.11.74

2. Goedert M, Spillantini MG, Del Tredici K, Braak H. 100 Years of Lewy pathology. Nat Rev Neurol (2012) 9:13-24. doi:10.1038/nrneurol.2012.242

3. Tanner CM, Goldman SM. Epidemiology of Parkinson's disease. Neurol Clin (1996) 14:317-35. doi:10.1016/S0733-8619(05)70259-0

4. Lhermitte J, Kraus WM, McAlpine D. Original papers: On the occurrence of abnormal deposits of iron in the brain in parkinsonism with special reference to its localisation. J Neurol Psychopathol (1924) 5:195-208. doi:10.1136/jnnp. s1-5.19.195

5. Berg D, Grote C, Rausch WD, Maurer M, Wesemann W, Riederer P, et al. Iron accumulation in the substantia nigra in rats visualized by ultrasound. Ultrasound Med Biol (1999) 25:901-4. doi:10.1016/S0301-5629(99)00046-0

6. Brar S, Henderson D, Schenck J, Zimmerman EA. Iron accumulation in the substantia nigra of patients with Alzheimer disease and parkinsonism. Arch Neurol (2009) 66:371-4. doi:10.1001/archneurol.2008.586

7. Jin L, Wang J, Jin H, Fei G, Zhang Y, Chen W, et al. Nigral iron deposition occurs across motor phenotypes of Parkinson's disease. Eur J Neurol (2012) 19:969-76. doi:10.1111/j.1468-1331.2011.03658.x

8. Jin L, Wang J, Zhao L, Jin H, Fei G, Zhang Y, et al. Decreased serum ceruloplasmin levels characteristically aggravate nigral iron deposition in Parkinson's disease. Brain (2011) 134:50-8. doi:10.1093/brain/awq319

9. Sofic E, Riederer P, Heinsen H, Beckmann H, Reynolds GP, Hebenstreit G, et al. Increased iron (III) and total iron content in post mortem substantia nigra of parkinsonian brain. J Neural Transm (1988) 74:199-205. doi:10.1007/ BF01244786

10. Wallis LI, Paley MN, Graham JM, Grunewald RA, Wignall EL, Joy HM, et al. MRI assessment of basal ganglia iron deposition in Parkinson's disease. J Magn Reson Imaging (2008) 28:1061-7. doi:10.1002/jmri.21563

11. Febbraro F, Giorgi M, Caldarola S, Loreni F, Romero-Ramos M. alpha-Synuclein expression is modulated at the translational level by iron. Neuroreport (2012) 23:576-80. doi:10.1097/WNR.0b013e328354alf0

12. Ostrerova-Golts N, Petrucelli L, Hardy J, Lee JM, Farer M, Wolozin B. The A53T alpha-synuclein mutation increases iron-dependent aggregation and toxicity. J Neurosci (2000) 20:6048-54.

13. Grolez G, Moreau C, Sablonniere B, Garcon G, Devedjian JC, Meguig S, et al. Ceruloplasmin activity and iron chelation treatment of patients with Parkinson's disease. BMCNeurol (2015) 15:74. doi:10.1186/s12883-015-0331-3

14. Ayton S, Lei P. Nigral iron elevation is an invariable feature of Parkinson's disease and is a sufficient cause of neurodegeneration. Biomed Res Int (2014) 2014:581256. doi:10.1155/2014/581256

15. Mounsey RB, Teismann P. Chelators in the treatment of iron accumulation in Parkinson's disease.IntJ CellBiol(2012)2012:983245.doi:10.1155/2012/983245

16. Dias V, Junn E, Mouradian MM. The role of oxidative stress in Parkinson's disease. J Parkinsons Dis (2013) 3:461-91. doi:10.3233/JPD-130230

17. Medeiros MS, Schumacher-Schuh A, Cardoso AM, Bochi GV, Baldissarelli J, Kegler A, et al. Iron and oxidative stress in Parkinson's disease: an observational study of injury biomarkers. PLoS One (2016) 11:e0146129. doi:10.1371/ journal.pone.0146129

18. Li W, Jiang H, Song N, Xie J. Oxidative stress partially contributes to iron-induced alpha-synuclein aggregation in SK-N-SH cells. Neurotox Res (2011) 19:435-42. doi:10.1007/s12640-010-9187-x

19. Beal MF, Oakes D, Shoulson I, Henchcliffe C, Galpern WR, Haas R, et al. A randomized clinical trial of high-dosage coenzyme Q10 in early Parkinson disease: no evidence of benefit. JAMA Neurol (2014) 71:543-52. doi:10.1001/ jamaneurol.2014.131

\section{FUNDING}

This work was supported by the National Natural Science Foundation of China (grant no. 81200973, 91332201) and by the National Key Research and Development Program Foundation of China (grant no. 2016YFC1306403).

20. Etminan M, Gill SS, Samii A. Intake of vitamin E, vitamin C, and carotenoids and the risk of Parkinson's disease: a meta-analysis. Lancet Neurol (2005) 4:362-5. doi:10.1016/S1474-4422(05)70097-1

21. Shoulson I. DATATOP: a decade of neuroprotective inquiry. Parkinson Study Group. Deprenyl and tocopherol antioxidative therapy of parkinsonism. Ann Neurol (1998) 44:S160-6. doi:10.1002/ana.410440724

22. Gavilan E, Pintado C, Gavilan MP, Daza P, Sanchez-Aguayo I, Castano A, et al. Age-related dysfunctions of the autophagy lysosomal pathway in hippocampal pyramidal neurons under proteasome stress. Neurobiol Aging (2015) 36:1953-63. doi:10.1016/j.neurobiolaging.2015.02.025

23. Jia G, Sowers JR. Autophagy: a housekeeper in cardiorenal metabolic health and disease. Biochim Biophys Acta (2015) 1852:219-24. doi:10.1016/j.bbadis. 2014.06.025

24. Metcalf DJ, Garcia-Arencibia M, Hochfeld WE, Rubinsztein DC. Autophagy and misfolded proteins in neurodegeneration. Exp Neurol (2012) 238:22-8. doi:10.1016/j.expneurol.2010.11.003

25. Lynch-Day MA, Mao K, Wang K, Zhao M, Klionsky DJ. The role of autophagy in Parkinson's disease. Cold Spring Harb Perspect Med (2012) 2:a009357. doi:10.1101/cshperspect.a009357

26. Scarffe LA, Stevens DA, Dawson VL, Dawson TM. Parkin and PINK1: much more than mitophagy. Trends Neurosci (2014) 37:315-24. doi:10.1016/j.tins. 2014.03.004

27. Xilouri M, Brekk OR, Stefanis L. Autophagy and alpha-synuclein: relevance to Parkinson's disease and related synucleopathies. Mov Disord (2016) 31:178-92. doi: $10.1002 / \mathrm{mds} .26477$

28. Cheung ZH, Ip NY. The emerging role of autophagy in Parkinson's disease. Mol Brain (2009) 2:29. doi:10.1186/1756-6606-2-29

29. Xu Y, Wang D, Luo Y, Li W, Shan Y, Tan X, et al. Beta amyloid-induced upregulation of death receptor 6 accelerates the toxic effect of $\mathrm{N}$-terminal fragment of amyloid precursor protein. Neurobiol Aging (2015) 36:157-68. doi:10.1016/ j.neurobiolaging.2014.07.027

30. Hong JH, Noh KM, Yoo YE, Choi SY, Park SY, Kim YH, et al. Iron promotes the survival and neurite extension of serum-starved PC12 cells in the presence of NGF by enhancing cell attachment. Mol Cells (2003) 15:10-9.

31. Lu Y, Yuan X, Sun Q, Ou Y. Autophagy activator promotes neuronal differentiation of adult adipose-derived stromal cells. Neural Regen Res (2013) 8:882-9. doi:10.3969/j.issn.1673-5374.2013.10.002

32. Smith ED, Prieto GA, Tong L, Sears-Kraxberger I, Rice JD, Steward O, et al. Rapamycin and interleukin-1beta impair brain-derived neurotrophic factordependent neuron survival by modulating autophagy. J Biol Chem (2014) 289:20615-29. doi:10.1074/jbc.M114.568659

33. Debbabi M, Nury T, Zarrouk A, Mekahli N, Bezine M, Sghaier R, et al. Protective effects of alpha-tocopherol, gamma-tocopherol and oleic acid, three compounds of olive oils, and no effect of Trolox, on 7-ketocholesterol-induced mitochondrial and peroxisomal dysfunction in microglial BV-2 cells. Int J Mol Sci (2016) 17:1973. doi:10.3390/ijms17121973

34. Hoover JL, Bond CE, Hoover DB, Defoe DM. Effect of neurturin deficiency on cholinergic and catecholaminergic innervation of the murine eye. Exp Eye Res (2014) 122:32-9. doi:10.1016/j.exer.2014.03.002

35. Wan W, Cao L, Liu L, Zhang C, Kalionis B, Tai X, et al. Abeta 1-42 $_{\text {oligomer- }}$ induced leakage in an in vitro blood-brain barrier model is associated with up-regulation of RAGE and metalloproteinases, and down-regulation of tight junction scaffold proteins. J Neurochem (2015) 134:382-93. doi:10.1111/ jnc. 13122

36. Wan W, Zhang C, Danielsen M, Li Q, Chen W, Chan Y, et al. EGb761 improves cognitive function and regulates inflammatory responses in the APP/PS1 mouse. Exp Gerontol (2016) 81:92-100. doi:10.1016/j.exger.2016. 05.007 
37. Liu J, Liu L, Xue Y, Meng F, Li S, Wang P, et al. Anti-neoplastic activity of lowdose endothelial-monocyte activating polypeptide-II results from defective autophagy and G2/M arrest mediated by PI3K/Akt/FoxO1 axis in human glioblastoma stem cells. Biochem Pharmacol (2014) 89:477-89. doi:10.1016/ j.bcp.2014.04.014

38. Pelizzoni I, Macco R, Morini MF, Zacchetti D, Grohovaz F, Codazzi F. Iron handling in hippocampal neurons: activity-dependent iron entry and mitochondria-mediated neurotoxicity. Aging Cell (2011) 10:172-83. doi:10.1111/j.1474-9726.2010.00652.x

39. Lan D, Wang W, Zhuang J, Zhao Z. Proteasome inhibitor-induced autophagy in PC12 cells overexpressing A53T mutant alpha-synuclein. Mol Med Rep (2015) 11:1655-60. doi:10.3892/mmr.2014.3011

40. Song JX, Lu JH, Liu LF, Chen LL, Durairajan SS, Yue Z, et al. HMGB1 is involved in autophagy inhibition caused by SNCA/alpha-synuclein overexpression: a process modulated by the natural autophagy inducer corynoxine B. Autophagy (2014) 10:144-54. doi:10.4161/auto.26751

41. Kiriyama Y, Nochi H. The function of autophagy in neurodegenerative diseases. Int J Mol Sci (2015) 16:26797-812. doi:10.3390/ijms161125990

42. Shi R, Weng J, Zhao L, Li XM, Gao TM, Kong J. Excessive autophagy contributes to neuron death in cerebral ischemia. CNS Neurosci Ther (2012) 18:250-60. doi:10.1111/j.1755-5949.2012.00295.x

43. Carew JS, Medina EC, Esquivel JA II, Mahalingam D, Swords R, Kelly K, et al. Autophagy inhibition enhances vorinostat-induced apoptosis via ubiquitinated protein accumulation. J Cell Mol Med (2010) 14:2448-59. doi:10.1111/j.1582-4934.2009.00832.x
44. Kizilarslanoglu MC, Ulger Z. Role of autophagy in the pathogenesis of Alzheimer disease. Turk J Med Sci (2015) 45:998-1003. doi:10.3906/sag1407-75

45. Baehrecke EH. Autophagy: dual roles in life and death? Nat Rev Mol Cell Biol (2005) 6:505-10. doi:10.1038/nrm1666

46. Cherra SJ III, Dagda RK, Chu CT. Review: autophagy and neurodegeneration: survival at a cost? Neuropathol Appl Neurobiol (2010) 36:125-32. doi:10.1111/j.1365-2990.2010.01062.x

47. Frake RA, Ricketts T, Menzies FM, Rubinsztein DC. Autophagy and neurodegeneration. J Clin Invest (2015) 125:65-74. doi:10.1172/JCI73944

48. Salminen A, Kaarniranta K, Kauppinen A, Ojala J, Haapasalo A, Soininen $\mathrm{H}$, et al. Impaired autophagy and APP processing in Alzheimer's disease: the potential role of Beclin 1 interactome. Prog Neurobiol (2013) 10(6-107):33-54. doi:10.1016/j.pneurobio.2013.06.002

Conflict of Interest Statement: The authors declare that the research was conducted in the absence of any commercial or financial relationships that could be construed as a potential conflict of interest.

Copyright (c) 2017 Wan, Jin, Wang, Wang, Fei, Ye, Pan, Wang and Zhong. This is an open-access article distributed under the terms of the Creative Commons Attribution License (CC BY). The use, distribution or reproduction in other forums is permitted, provided the original author(s) or licensor are credited and that the original publication in this journal is cited, in accordance with accepted academic practice. No use, distribution or reproduction is permitted which does not comply with these terms. 